\title{
EL USO DE LAS TIC'S EN TRÁMITES DE ADJUDICACIÓN DE APOYO Y CONCILIACIOON FRENTE AL COVID-19
}

The use of the TIC'S in support award and conciliation procedures against COVID-19

\author{
Mariana Pinto-Barriga \\ mapintob2@poligran.edu.co \\ Anlly Adriana Rodríguez-Toro \\ aarodriguezt@poligran.edu.co
}

Institución Universitaria Politécnico Grancolombiano

Derecho y Psicología

Colombia

\section{RESUMEN}

Con el fin de indagar acerca de los mecanismos alternativos de solución de conflictos, en especial la conciliación, que son instrumentos diseñados para descongestionar el sistema judicial colombiano a través de la Ley 640 de 2001, se pretende desarrollar el mecanismo de la conciliación en específico y su actual importancia con la implementación de la Ley 1996 de 2019, la cual busca regular la capacidad de los colombianos que presentan alguna condición de discapacidad, eliminado el antiguo proceso de interdicción y reemplazándolo por los trámites de adjudicación de apoyos, los cuales pueden realizarse por los centros de conciliación.

Se busca realizar el estudio de la respectiva aplicación de esta, teniendo en cuenta el obstáculo que generó la actual pandemia causada por COVID-19, por medio del método cualitativo descriptivo, con el fin de descubrir las problemáticas presentadas en el acceso a la justicia para la población que presenta alguna condición de discapacidad y brindar posibles soluciones desde el ámbito académico.

\section{PALABRAS CLAVE:}

Personas en condición de discapacidad, apoyo, Tecnologías de la Información y la Comunicación (TIC), mecanismos alternativos de solución de conflictos, conciliación

\section{INTRODUCCIÓN}

Con el fin de cumplir las obligaciones del Estado, el Congreso de la República de Colombia, en concordancia con el artículo 116 inciso 4 (Const., 1991), y con la ley estatutaria de administración de justicia (Ley 270, 1996), dicta los principios de la administración de justicia, y promueve los MASC, incorporando, más adelante en el estatuto, el cual define la conciliación (Decreto 1818, 1998, art. 1).

Por otro lado, en medio de la crisis sanitaria que se presenta en Colombia a raíz del COVID-19, el sistema judicial tuvo que suspender la presencialidad y adaptarse a las TIC para seguir brindando el acceso a la administración de justicia. Esta situación no fue ajena a las conciliaciones extrajudiciales, las cuales se están adelantando en escenarios virtuales.

Una de las novedades que aborda el presente artículo es la adjudicación de apoyos a las personas en condición de discapacidad, (Ley 1996, 2019, art. 17, Inc. 2), proceso que debido a la contingencia también se vio suspendido. En cumplimiento de parámetros internacionales, se han expedido diferentes normas, buscando garantizar a la población

\section{ENCUENTRO CON SEMILLEROS, APORTES Y REFLEXIONES \\ Volumen 2 No 2 (2020) | ISSN 2711-4414}


con alguna condición de discapacidad cinco ámbitos vitales, como lo son "salud, educación, empleo, transporte y accesibilidad” (Martínez-Rozo, Uribe-Rodríguez, \& Velázquez-González, 2015, p. 49).

¿Cuáles son los limitantes que se presentan para el administrador de justicia, su equipo psicosocial y las personas en condición de discapacidad, en los escenarios de conciliación y adjudicación de apoyos con uso de las TIC, ante la situación derivada de la pandemia?

La metodología que se plantea consiste en una investigación cualitativa, mediante la implementación de diferentes herramientas, de revisión de fuentes bibliográficas, bases de datos como Sistema de Información de la Conciliación, el Arbitraje y la Amigable Composición (SICAAC), doctrina, jurisprudencia relevante y norma vigente, con aplicación de un método deductivo comparado, que permita generar unas recomendaciones necesarias para garantizar un debido proceso, eficiencia y eficacia en los entornos de conciliación y adjudicaciones de apoyo.

Desde el marco jurídico analítico se encontraron relevantes las leyes, como la Ley 1996 de 2019, "por medio de la cual se establece el régimen para el ejercicio de la capacidad legal de las personas con discapacidad mayores de edad", 26 de agosto de 2019, D.O. No. 51057. El código civil Colombiano [COP] libro cuarto, Ley 84 de 1873, 31 de mayo de 1873 (Colombia), "por el cual se modifican normas relativas a la conciliación y se dictan otras disposiciones".

En cuanto la Ley 1346 de 2009, "por medio de la cual se aprueba la Convención sobre los derechos de las personas con discapacidad adoptada por la Asamblea General de las Naciones Unidas el 13 de diciembre de 2006", 31 de julio de 2009, D.O. No. 47.427. Por otra parte, la Ley 1618 de 2013, "por medio de la cual se establecen las disposiciones para garantizar el pleno ejercicio de los derechos de las personas con discapacidad", 27 de febrero de 2013, D.O. No. 48.417. Seguido encontramos la Ley 1680 de 2013, "por la cual se garantiza a las personas ciegas y con baja visión, el acceso a la información, a las comunicaciones, al conocimiento y a las tecnologías de la información y de las comunicaciones", 20 de noviembre de 2013, D.O. No. 48.980. Posterior, la Ley 1306 de 2009, "por la cual se dictan normas para la protección de personas con discapacidad mental y se establece un régimen de la representación legal de incapaces emancipados", 5 de junio del 2009, D.O. No. 47.371.

\section{Objetivos}

1. Analizar la necesidad del equipo psicosocial en la conciliación con la utilización de herramientas tecnológicas.

2. Investigar la estadística a nivel nacional de las conciliaciones efectivas y su procedimiento para los colombianos en condición de discapacidad.

3. Reconocer cuáles son los mecanismos tecnológicos utilizados en la conciliación y su aplicación en medio de la crisis sanitaria actual.

\section{MÉTODO}

Mediante un enfoque cualitativo descriptivo por método deductivo comparado, con aplicación de análisis de la estadística de conciliaciones extrajudiciales en Bogotá de 2016 a 2019 en el SICAAC, contiene la estadística a nivel nacional desde el año 2016 a 2019, de los diferentes mecanismos, y su implementación por los ciudadanos; entre los filtros que reposan en el sistema se encuentran los factores de edad, estrato socioeconómico, nivel de escolaridad, área consultada y resultado obtenido.

El semillero estudia la siguiente información con la finalidad de identificar el impacto de este mecanismo en la ciudad de Bogotá, del año 2016 a 2019. Consultando otras fuentes, tales como el DNP, o la página oficial de Discapacidad Colombia, se evidencia que no se tiene estadística que sirva para la presente investigación, y el uso de los diferentes mecanismos complementados antes y después de la pandemia.

Como resultados se obtuvieron los siguientes datos: entre los años 2016 y 2019 las personas entre los 36 y 40 años fueron las que más frecuentaban a las conciliaciones, con un total de 26.094 convocantes, por tres años consecutivos. De los 
resultados obtenidos, según los convocantes por estrato, se tiene que, durante los cuatro años analizados, el estrato 2 fue el mayor convocante, con un total de 126.570 solicitudes, sin embargo, se evidencia que durante los años 2018 y 2019 la mayor frecuencia de convocantes no informaron su estrato.

Con relación al grado de escolaridad por año, los convocantes que dieron un mayor uso al mecanismo de la conciliación no informaron su grado, sin embargo, hay una variación entre los grados de educación media o secundaria alta, quienes fueron los convocantes más frecuentes del año 2016, del grado educación básica secundaria, quienes recurrieron más en los años 2017 y 2018, y los mismos quienes en el 2019 tuvieron la misma cantidad de convocantes.

Se evidencia de igual forma, con relación a los resultados de dichas solicitudes, que el número total de actas de conciliación tuvo altibajos entre los cuatro años analizados, sin embargo, presentó un alza considerable en el 2019, cuyo total fue de 22.756 actas registradas, las constancias de inasistencia convocado, a partir del año 2017, se mantuvieron en el rango de 13.118 cantidades registradas y las constancias de no acuerdo se fueron incrementando en un mínimo cada año, obteniendo así, en el 2019, 10.689 cantidades registradas.

El área en la que más se presentaron solicitudes fue la de civil y comercial, donde en el 2019 se obtuvo el mayor resultado con un total de 80.790 cantidades registradas; en segundo lugar, las solicitudes más frecuentes son en el área de familia. Después de analizar las encuestas realizadas por el SICAAC, se puede evidenciar que no hay una estadística que nos demuestre cuántas personas que poseen algún tipo discapacidad fueron partícipes del proceso de conciliación, ni qué clase de limitación funcional presentan; es una población que debe tener un registro, porque el trámite de sus procesos es totalmente diferente. Sin estos datos no se logra establecer el alcance de las herramientas dispuestas para su atención integral.

\section{DISCUSIÓN Y CONCLUSIÓN}

A pesar de los lineamientos impartidos para garantizar un correcto desarrollo en sus derechos y deberes constitucionales, debido a la pandemia de forma presencial y virtual, los mismos no son garantes de la salubridad de los funcionarios.

Si bien en las conciliaciones judiciales, la norma en materia civil y de familia contempla la conformación de un equipo psicosocial, brindando la adjudicación de apoyos; no existe una imposición en este sentido para centros públicos o privados en conciliaciones extrajudiciales y otorgamiento de apoyos voluntarios.

La normativa para garantizar el acceso a la administración de justicia para la población con condiciones de discapacidad de diferente índole se encuentra ampliamente definidos en las normas vigentes, sin embargo, no existe una entidad estatal que lleve la estadística del uso de estas herramientas, que permita evidenciar el alcance de dichos mecanismos, ni generar investigación cualitativa, se recomienda agregar dichos parámetros a la estadística ya existente en el SICAAC. No existe una unificación de criterios frente al manejo de las audiencias de conciliación extrajudiciales en época de pandemia, ni de adjudicación de apoyos, por lo que muchos centros públicos y privados se negaron a prestar el servicio. Incluso antes de la contingencia ya existía el uso de las TIC con el fin de proteger el derecho del artículo 229 de la Constitución, sin embargo, estos no son de conocimiento público, por lo que su falta de divulgación puede constituir una barrera.

La interdisciplinariedad con áreas como psicología no se encuentra debidamente soportada, ni en las conciliaciones ni en la adjudicación de apoyo en escenarios digitales, que revisten igual importancia, pues el conciliador no cuenta con las aptitudes ni actitudes requeridas para trabajar diferentes tipos de discapacidad, que garantice que no se violenten los derechos de las personas con condición de discapacidad. 


\section{RESULTADOS}

El equipo interdisciplinario es esencial al momento de realizar las conciliaciones, ya que con son los psicólogos y trabajadores sociales quienes se encuentran capacitados para tratar la parte emocional de las personas, y además pueden llegar a servir en caso de una crisis durante el proceso como también puedan colaborar y fungir como una herramienta para lograr una comunicación inequívoca de la voluntad del titular del acto.

EI SICAA, ente encargado de las cifras y estadísticas de las conciliaciones a nivel nacional, brinda información respecto a las conciliaciones a nivel nacional, y categoriza las mismas por estrato, género, asunto y edad, sin embargo, no presenta una cifra o estadística de la población con condiciones de discapacidad que se presentan a estos actos, a pesar de que estas cifras son necesarias para que los centros de conciliación, ya sean privados o públicos, realicen los ajustes razonables y apoyo a esta población.

También se pudo evidenciar la falta de formación de los funcionarios para brindar atención a la población que presenta alguna condición de discapacidad, ya que la atención en ocasiones es vacía o simplemente evadida por desconocimiento de la norma o de las barreras de comunicación. Es así como la rama judicial debe adaptarse a la tecnología y a los actos jurídicos por estos medios, utilizando aplicativos y otras herramientas, pues a pesar de que muchos centros cuentan con estas herramientas, el personal no está capacitado para su uso y dichas personas desconocen estas herramientas.

Además, las limitantes para el administrador de justicia se ven reflejadas al analizar las encuestas realizadas por el SICAAC se puede evidenciar que no hay una estadística que nos demuestre cuántas personas que presentan alguna condición de discapacidad fueron participes en conciliaciones, ni qué limitante funcional poseen; es una población que debe tener un registro porque el trámite de sus procesos es totalmente diferente, y sin estos datos no se logra establecer el alcance de las herramientas dispuestas para su atención integral, ni el alcance de las mismas.

\section{REFERENCIAS BIBLIOGRÁFICAS}

Centro de Arbitraje y Conciliación. Cámara de Comercio de Bogotá. (2020). Circular 001 del 16 de marzo de 2020. Recuperado de https://www.cerlatam.com/covid19/normas-regionales-de-colombia/circular-001-de-2020camara-de-comercio-de-bogotal

Código General del Proceso [Código]. (2012). Secretaría del Senado.

Congreso de Colombia. (15 de marzo de 1996). Ley estatutaria de la administración de justicia [Ley 270 de 1996]. DO: 42.745

Congreso de Colombia. (24 de enero de 2001). Por la cual se modifican normas relativas a la conciliación y se dictan otras disposiciones. [Ley 640 de 2001]. DO: 44.303

Congreso de Colombia. (10 de julio de 2007). Organiza el Sistema Nacional de Discapacidad y se dictan otras disposiciones. [Ley 1145 de 2007]. DO: 46.685

Congreso de Colombia. (22 de enero de 2009). Reforma la Ley 270 de 1996 Estatutaria de la Administración de Justicia. [Ley 1285 de 2009]. DO: 47.240

Congreso de Colombia. (31 de julio de 2009). Aprueba la "Convención sobre los Derechos de las personas con Discapacidad”, adoptada por la Asamblea General de la Naciones Unidas el 13 de diciembre de 2006. [Ley 1346 de 2009]. DO: 47.427 
Congreso de Colombia. (26 de agosto de 2019). Régimen para el ejercicio de la capacidad legal de las personas con discapacidad mayores de edad [Ley 1996 de 2019]. DO: 51.057

Congreso de Colombia. (27 de febrero de 2013). Se establecen las disposiciones para garantizar el pleno ejercicio de los derechos de las personas con discapacidad. [Ley Estatutaria 1618 de 2013]. DO: 48.717

Consejo Superior de la Judicatura. (Acuerdo PCSJA20-11517 de 15 de marzo de 2020). Recuperado de: http://actosadministrativos.ramajudicial.gov.co/GetFile.ashx?url=\%7E\%2FApp_Data\%2FUpload\%2FPCSJA2O11517.pdf

Consejo Superior de la Judicatura. (Acuerdo PCSJA20-11597 de 15 de julio de 2020). Recuperado de: http://actosadministrativos.ramajudicial.gov.co/GetFile.ashx?url=\%7E\%2FApp_Data\%2FUpload\%2FPCSJA2O11597.pdf

Constitución Política de Colombia. (1991). Bogotá: Ed. Legis.

Corte Constitucional. (21 de octubre de 2011). Sentencia T-799 [MP Humberto Antonio Sierra Porto]

Corte Constitucional. (19 de febrero de 1998). Sentencia C-037 [MP Jorge Arango Mejía]

Departamento Administrativo Nacional de Estadística, DANE. (2018). Censo Nacional de Población

y Vivienda 2018. Recuperado de: https://www.dane.gov.co/index.php/estadisticas-por-tema/demografia-ypoblacion/censo-nacional-de-poblacion-y-vivenda-2018/cuantos-somos

Gaitán, J., \& Rodríguez, J. (2020). Investigación Socio-Humanística del Derecho. La conciliación extrajudicial en tiempos de COVID - 19. [144-167] Recuperado de:

https://repository.usta.edu.co/bitstream/handle/11634/27409/La\%20Conciliaci\%C3\%B3n\%20Extrajudicial\%20En\%2 oTiempos\%20De\%20Covid-19.pdf?sequence=1\&isAllowed=y

Gobierno Nacional. Ministerio de Justicia y del Derecho. (1998). Estatuto de los mecanismos alternativos de solución de conflictos [Decreto 1818 de 1998]. DO: 43.380

Gobierno Nacional, Ministerio de Justicia y del Derecho. (28 de marzo de 2020). Por el cual se adoptan medidas de urgencia para garantizar la atención y la prestación de los servicios por parte de las autoridades públicas y los particulares que cumplan funciones públicas y se toman medidas para la protección laboral y de los contratistas de prestación de servicios de las entidades públicas, en el marco del Estado de Emergencia Económica, Social y Ecológica [Decreto Legislativo 491 de 2020]. DO: 51.270

Gobierno Nacional, Ministerio de Justicia y del Derecho. (22 de marzo de 2020). Por el cual se dictan medidas el servicio a cargo de las comisarías de familia, dentro del Estado de Emergencia Económica, Social y Ecológica [Decreto Legislativo 460 de 2020]. DO: 51.264

Ministerio de Salud de Colombia. (2020). Coronavirus Covid-19. Recuperado de: https://covid19.minsalud.gov.co/

Martínez-Rozo, A., Uribe-Rodríguez, A., \& Velázquez-González, H. (2015). La discapacidad y su estado actual en la legislación colombiana. Duazary, 12(1), 49 - 58. Recuperado de: https://doi.org/10.21676/2389783X.1398

Ministerio de Justicia y del Derecho. (2020). Subdirección de Gestión de Información en Jurídica. Recuperado de: https://sej.minjusticia.gov.co/RamaJudicial/Paginas/Introduccion.aspx\#: :text=La\%20rama\%20judicial\%20del\%2 opoder, para\%2ogarantizar\%20una\%20convivencia\%20pac\%C3\%ADfica. 
Ministerio de Justicia y del Derecho. (s.f.) Guía de atención a las personas con discapacidad en el acceso a la justicia. Recuperado de:

http://discapacidadcolombia.com/phocadownloadpap/GOBIERNO/GUIA\%20DISCAPACIDAD\%20ACCESO\%20A\%2 OLA\%20JUSTICIA.pdf

Procuraduría General de la Nación. (2020). ABC de la conciliación extrajudicial administrativa durante la emergencia sanitaria. Recuperado de:

https://www.procuraduria.gov.co/portal/media/file/CARTILLA\%20ABC\%20DE\%20LA\%20CONCILIACIO\%CC\%81N\%2 OEXTRAJUDICIAL\%20ADMINISTRATIVA\%20DURANTE\%20EL\%20ESTADO\%20DE\%20EMERGENCIA\%20.pdf 